\title{
Genetic Analysis and Some Properties of Starch in Waxy Mutant Wheat Tanikei A6599-4
}

\author{
Chikako Kiribuchi-Otobe*, Takashi Yanagisawa and Hisashi Yoshida \\ National Institute of Crop Science, 2-1-18 Kannondai, Tsukuba, Ibaraki 305-8518, Japan
}

\begin{abstract}
Waxy mutant wheat Tanikei A6599-4, which was induced from Tanikei A6099 (low-amylose line), exhibited a unique pasting curve with stable hot paste viscosity in the Rapid ViscoAnalyser (RVA) measurement. Analysis of the amylose content of reciprocal $F_{1}$ seeds revealed the incomplete dominance and gene dosage effect in hexaploid wheat. In the dominance among multiple $W x-D 1$ alleles, the $W x-D 1$ allele of Tanikei A6099 partially dominated that of Tanikei A6599-4, and the allele of Tanikei A6599-4 partially dominated that of Tanikei H1881 (waxy line of amylose-free type). Genetic analysis using $\mathrm{DH}_{2}$ lines suggested that both the waxy character and stable hot paste viscosity of Tanikei A6599-4 are controlled by the same mutated $W x-D I$ gene. We studied the pasting properties of Tanikei A6599-4 starch and compared them to those of other starches. At a low suspension concentration (6\%), the peak viscosity of Tanikei A6599-4 was closer to that of Tanikei H1881 but the peak viscosity temperature was the same as that of Norin 61 (nonwaxy line). Addition of $\mathrm{NaCl}$ did not affect the starch pasting properties of Tanikei A6599-4, while potato starch which shows a more stable hot paste viscosity than cereal starches, was significantly affected by the $\mathrm{NaCl}$ treatment.
\end{abstract}

Key Words: Triticum aestivum, waxy, amylose content, Rapid ViscoAnalyser, pasting property.

\section{Introduction}

Amylose synthesis in the cereal grain endosperm is mainly controlled by the granule-bound starch synthase known as the Wx protein (Echt and Schwartz 1981, Sano 1984, Rohde et al. 1988). Endosperm starch generally consists of amylose and amylopectin, but in the mutant lacking the $\mathrm{Wx}_{\mathrm{x}}$ protein, endosperm starch consists exclusively of amylopectin and is referred to as "waxy starch." Although the waxy endosperm has been identified in several crops (Eriksson 1969), it was not found in hexaploid wheats, possibly because of the triplication of $W x$ genes ( $W x-A I$, $W x-B I, W x-D I)$ coding for $\mathrm{Wx}$ protein isoforms (Wx-Al,

Communicated by C. Kaneda

Received January 10, 2001. Accepted June 6, 2001.

*Corresponding author (e-mail: ochika@affrc.go.jp)
Wx-B1, Wx-D1) (Chao et al. 1989, Nakamura et al. 1993). The first waxy hexaploid wheats were obtained in Japan from 3 different sources: (1) cross combination between Kanto 107 and Bai Huo (Nakamura et al. 1995), (2) cross combination between Saikai 168 and Tanikei A6099 (Kiribuchi-Otobe et al. 1997), and (3) mutation from Kanto 107 (Yasui et al. 1997). These waxy lines showed similar characteristics; amylose content was almost zero (amylosefree), pollen grains and endosperm starch granules stained red-brown with a potassium iodide and iodine solution, and no Wx proteins were observed in the endosperm (Nakamura et al. 1995, Kiribuchi-Otobe et al. 1997, Yasui et al. 1997). They were referred to as "amylose-free", based on the interpretation of Nakamura et al. (1995). Allelism test conducted by pollen analysis showed that they had null alleles at $3 W x$ loci (Kiribuchi-Otobe et al. 1998a).

We obtained a different type of waxy wheat, Tanikei A6599-4, by sodium azide treatment (Kiribuchi-Otobe $e t a l$. 1998b). Analysis by amperometric titration showed that the line contained $1.6 \%$ amylose and its pollen grains and endosperm starch granules stained dark brown with a potassium iodide and iodine solution. The line contained the same amount of Wx protein as its parental line Tanikei A6099 (Yanagisawa et al. 2001). Rapid ViscoAnalyser (RVA) measurement showed that the peak viscosity temperature was higher and the hot paste viscosity was more stable in Tanikei A6599-4 than in amylose-free wheat (KiribuchiOtobe et al. 1998b). Although pollen analysis suggested that the waxy character of Tanikei A6599-4 was due to a mutation occurring at the Wx-Dl locus (Kiribuchi-Otobe et al. 1998b), certain aspects remained to be analyzed, e.g., dominance among multiple $W x-D 1$ alleles and whether the stable hot paste viscosity in Tanikei A6599-4 was controlled by the same mutated $W x-D I$ gene responsible for its waxy character. We studied the mode of inheritance of the waxy character in detail and provide information on the unique pasting properties of Tanikei A6599-4 and their genesis.

\section{Materials and Methods}

Determination of apparent amylose content of $F_{l}$ seeds. Materials studied included Norin 61 (normal amylose), Tanikei A6099 (low-amylose), Tanikei H1881 (waxy: amylose-free), and Tanikei A6599-4 (waxy: a small amount of amylose). Norin 61 is a standard cultivar with $3 \mathrm{Wx}$ protein isoforms. Tanikei A6099 is a low-amylose mutant line induced from Kanto 107 by EMS treatment (Oda et al. 
1992). Both Tanikei A6099 and Kanto 107 lack Wx-A1 and $\mathrm{Wx}-\mathrm{B} 1$ proteins, and the amount of the $\mathrm{Wx}-\mathrm{D} 1$ protein in Tanikei A6099 is lower than that in Kanto 107 (Yanagisawa et al. 1996). Tanikei $\mathrm{H} 1881$ is a waxy wheat of amylosefree type derived from a cross combination between Saikai 168 and Tanikei A6099 (Kiribuchi-Otobe et al. 1997). Tanikei A6599-4 is a waxy mutant line induced from Tanikei A6099 by sodium azide treatment (Kiribuchi-Otobe et al.). In 1995, Tanikei H1881 was reciprocally crossed with Norin 61 and Tanikei A6099 to yield $F_{1}$ seeds. In 1996, Tanikei A6599-4 was reciprocally crossed with Tanikei A6099 and Tanikei H1881 to yield $F_{1}$ seeds. Self-pollinated seeds were also prepared. In all the cases, central florets were removed and the number of florets per spike was adjusted to 20 and their awns were cut off to equalize the grain filling conditions. Using a motor drill, flour was obtained from the endosperm of each seed. The apparent amylose content of the flour was colorimetrically determined using a Technicon Autoanalyser as described elsewhere (Oda et al. 1992).

\section{Inheritance of stable hot paste viscosity}

Kanto 118 and Tanikei A6599-4 were used as parents. Kanto 118 , an offspring of Kanto 107 , is a low-amylose line in which only the $W x-D I$ gene produces the $W x$ protein. We obtained 22 doubled haploid (DH) lines by haploid breeding using the maize technique (Suenaga and Nakajima 1989, Ushiyama et al. 1991) from $\mathrm{F}_{1}$ hybrids between Kanto 118 and Tanikei A6599-4. Grains of all the $\mathrm{DH}_{2}$ lines and their parents were milled into flour using a Brabendor Quadrumat Jr. mill. Grains of Tanikei H1881 were also milled into flour. Four gram of flour was mixed with $25 \mathrm{ml}$ of $0.01 \mathrm{M}$ silver nitrate $\left(\mathrm{AgNO}_{3}\right)$ solution and the paste viscosity was measured using the RVA. $\mathrm{AgNO}_{3}$ was used as an inhibitor of $\alpha$-amylase in flour. The suspension was heated from 34 to $94^{\circ} \mathrm{C}$ at $5^{\circ} \mathrm{C} / \mathrm{min}$ and held at $94^{\circ} \mathrm{C}$ for $5 \mathrm{~min}$, then cooled to $34^{\circ} \mathrm{C}$ at $5^{\circ} \mathrm{C} / \mathrm{min}$.

\section{Starch pasting properties}

Starches of Norin 61, Tanikei H1881, and Tanikei A6599-4 were isolated by washing with water and centrifugation as described elsewhere (Kiribuchi-Otobe et al. 1998b). Potato starch was purchased from Wako Pure Chemical Industries, Ltd. To evaluate the concentration effect, 1.5, 2.25 , and $3 \mathrm{~g}$ of wheat starch were mixed with $25 \mathrm{ml}$ of distilled water to prepare $6 \%, 9 \%$ and $12 \%$ suspensions. To evaluate the effect of sodium chloride $(\mathrm{NaCl}), 3 \mathrm{~g}$ of wheat starch and $2 \mathrm{~g}$ of potato starch were suspended in $25 \mathrm{ml}$ of distilled water or $0.08 \% \mathrm{NaCl}$ solution. The pasting viscosity of the suspension was measured using the RVA as described above.

\section{Results}

Determination of apparent amylose content of $F_{1}$ seeds.

The amylose content of the endosperm flour of $F_{1}$ seeds and their parents is shown in Table 1. Since the flour amylose content was determined colorimetrically and the contribution of amylopectin to the absorbance was not considered, the waxy lines showed a higher apparent amylose content than their actual content. In our previous paper where the same Technicon Autoanalyser was used, we reported that the amylose content of Norin 61 and Tanikei A6099 (previously designated as $\mathbf{M}_{3}-84$ ) was about 30 and $15 \%$ (Oda et al. 1992). Compared to these values, the present values were lower by $2-4 \%$. The removal of central

Table 1. Apparent amylose content of reciprocal $F_{1}$ seeds

\begin{tabular}{|c|c|c|c|c|c|}
\hline \multirow{2}{*}{ Year } & \multirow{2}{*}{ Cross combination } & \multicolumn{3}{|c|}{$W x$ gene dosage } & \multirow{2}{*}{$\begin{array}{l}\text { Apparent amylose content } \\
(\%)\end{array}$} \\
\hline & & $W x-A I$ & $W x-B I$ & $W x-D I$ & \\
\hline \multirow[t]{7}{*}{1995} & Norin 61 (self) & 3 & 3 & 3 & $25.0 \pm 0.9 \mathrm{a}$ \\
\hline & Tanikei A6099 (self) & 0 & 0 & 3 & $13.1 \pm 1.5 \mathrm{~d}$ \\
\hline & Tanikei H1881 (self) & 0 & 0 & 0 & $2.2 \pm 0.6 \mathrm{~g}$ \\
\hline & Norin61/Tanikei H1881 & 2 & 2 & 2 & $22.1 \pm 1.5 b$ \\
\hline & Tanikei H1881 / Norin 61 & 1 & 1 & 1 & $17.5 \pm 2.3 \mathrm{c}$ \\
\hline & Tanikei A6099/Tanikei H1881 & 0 & 0 & 2 & $8.5 \pm 0.6 \mathrm{e}$ \\
\hline & Tanikei H1881/Tanikei A6099 & 0 & 0 & 1 & $5.0 \pm 0.4 \mathrm{f}$ \\
\hline \multirow[t]{7}{*}{1996} & Tanikei A6099 (self) & 0 & 0 & 3 & $12.4 \pm 0.9 \mathrm{a}$ \\
\hline & Tanikei H1881 (self) & 0 & 0 & 0 & $2.1 \pm 0.1 \mathrm{~g}$ \\
\hline & Tanikei A6599-4 (self) & 0 & 0 & $\stackrel{3}{=}$ & $3.7 \pm 0.4 \mathrm{~d}$ \\
\hline & Tanikei A6099/Tanikei A6599-4 & 0 & 0 & $\underline{2}+\underline{1}$ & $8.7 \pm 0.6 b$ \\
\hline & Tanikei A6599-4/Tanikei A6099 & 0 & 0 & $\underline{1}+\underline{\overline{2}}$ & $6.3 \pm 0.4 \mathrm{c}$ \\
\hline & Tanikei H1881/Tanikei A6599-4 & 0 & 0 & 1 & $2.7 \pm 0.1 \mathrm{f}$ \\
\hline & Tanikei A6599-4/Tanikei H1881 & 0 & 0 & 2 & $3.1 \pm 0.2 \mathrm{e}$ \\
\hline
\end{tabular}

Values followed by different letters in the same year differ significantly based on the LSD method at $\mathrm{P}=0.05$.

Underlined parts in the $W x-D 1$ row indicate mutated genes (- Tanikei A6099, = Tanikei A6599-4). 
florets and cutting of awns probably modified the ripening conditions and affected the amylose content but not the order relation. $F_{1}$ seeds showed values intermediate between those of the parents in all the cross combinations (Table 1), indicating that the gene of the line with a higher amylose content was partially dominant over that of the line with a lower content. The amylose content differed between reciprocal $F_{1}$ seeds. When the line with a lower amylose content was used as a maternal parent, $F_{1}$ seeds showed a lower amylose content than when it was used as a pollen parent. These results suggest the presence of a gene dosage effect, because the endosperm had 1 dose of the paternal and 2 doses of maternal allele due to double fertilization.

\section{Inheritance of stable hot paste viscosity}

Twenty-two $\mathrm{DH}_{2}$ lines segregated into waxy 11: nonwaxy 11 , indicating a good fit to the expected $1: 1$ ratio. To determine the stability of hot paste viscosity, we selected the peak viscosity temperature and time maintained above $80 \%$ of peak viscosity (Fig. 1). All the waxy lines showed a pasting curve similar to that of Tanikei A6599-4, and none of the nonwaxy lines exhibited a stable hot paste viscosity (Fig. 2). Tanikei H1881 was plotted in a separate area. These results suggest that both the waxy character and stable hot paste viscosity of Tanikei A6599-4 were controlled by the same gene, which must be $W x-D 1$, since the waxy character of Tanikei A6599-4 was ascribed to the mutation at the Wx-DI locus (Kiribuchi-Otobe et al. 1998b).

\section{Starch pasting properties}

In the pasting curves of 3 cultivars with different starch concentrations (Fig. 3), the peak viscosity dropped as the concentration decreased in the all cases. The behavior of the peak viscosity temperature was noteworthy. The peak viscosity temperature of Norin 61 starch was constant at $94^{\circ} \mathrm{C}$ (maximum temperature), while waxy wheat starch (Tanikei H1881 and Tanikei A6599-4) showed a higher value for the peak viscosity temperature as the concentration decreased. When the 2 types of waxy wheat were compared, the in-

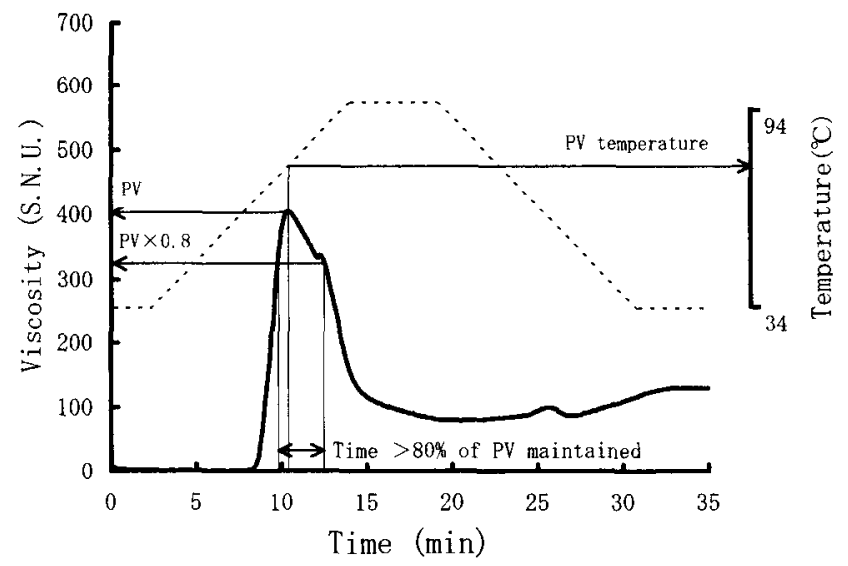

Fig. 1. Flour pasting curves in RVA measurement PV: Peak viscosity.

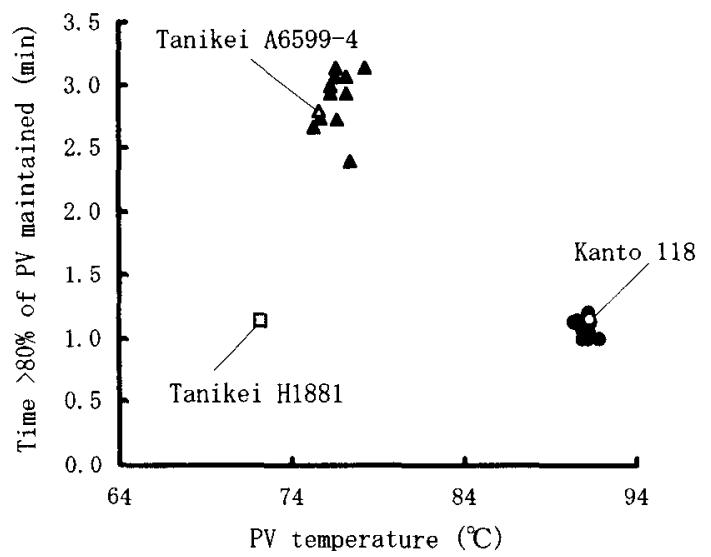

Fig. 2. Stability of hot paste viscosity of $\mathrm{DH}_{2}$ lines. : Nonwaxy, $\mathbf{A}$ : Waxy (Tanikei A6599-4 type) PV: Peak viscosity.

crease in Tanikei A6599-4 $\left(72.5^{\circ} \mathrm{C} \rightarrow 90.5^{\circ} \mathrm{C} \rightarrow 94^{\circ} \mathrm{C}\right)$ was larger than that in Tanikei $\mathrm{H} 1881\left(68.5^{\circ} \mathrm{C} \rightarrow 70.5^{\circ} \mathrm{C} \rightarrow\right.$ $75.5^{\circ} \mathrm{C}$ ).

The effect of $\mathrm{NaCl}$ on the starch pasting properties
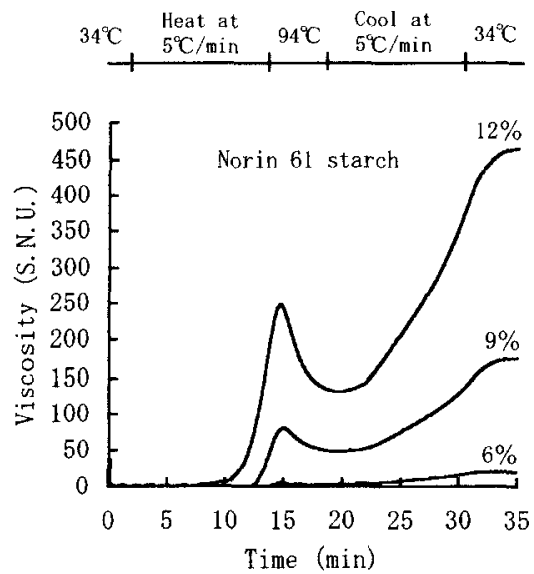
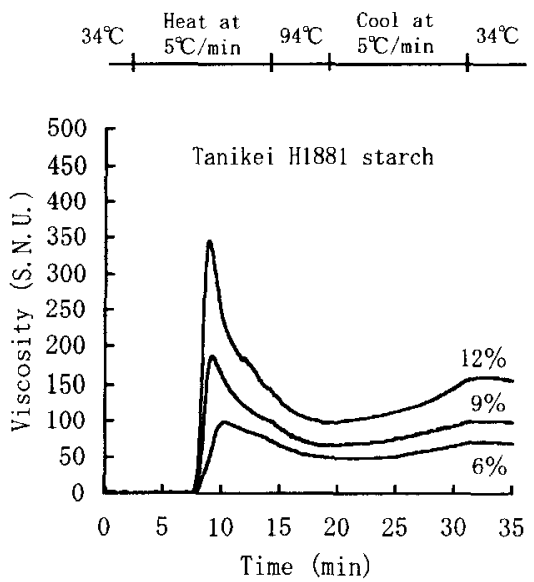
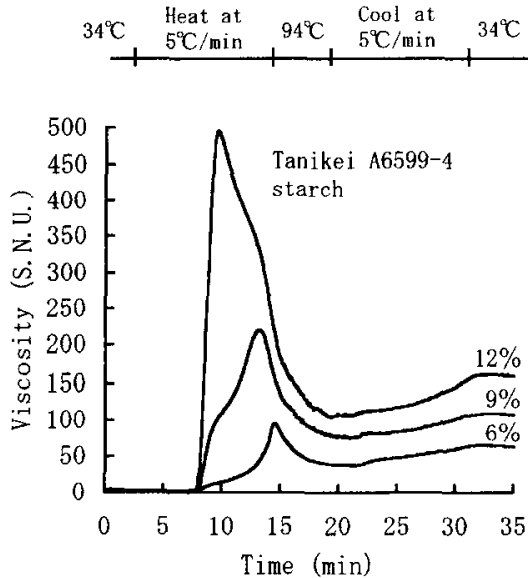

Fig. 3. Effect of starch concentration on pasting properties. 
$34^{\circ} \mathrm{C}$, Heat at $5^{\circ} \mathrm{C} / \mathrm{min}, 94^{\circ} \mathrm{C}, \mathrm{Cool}$ at $5^{\circ} \mathrm{C} / \mathrm{min}, 34^{\circ} \mathrm{C}$

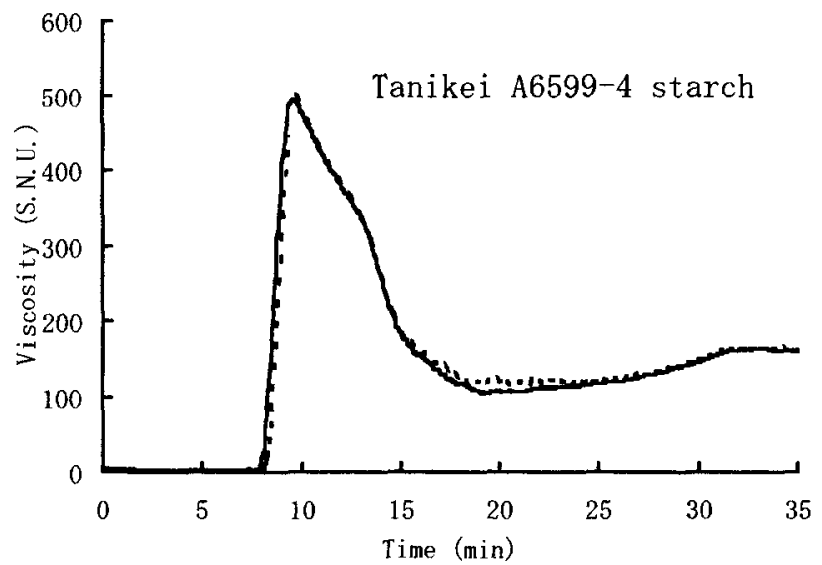

$34^{\circ} \mathrm{C}$, Heat at $55^{\circ} \mathrm{C} / \mathrm{min}, 94^{\circ} \mathrm{C}, \mathrm{Cool}$ at $5^{\circ} \mathrm{C} / \mathrm{min}, 34^{\circ} \mathrm{C}$

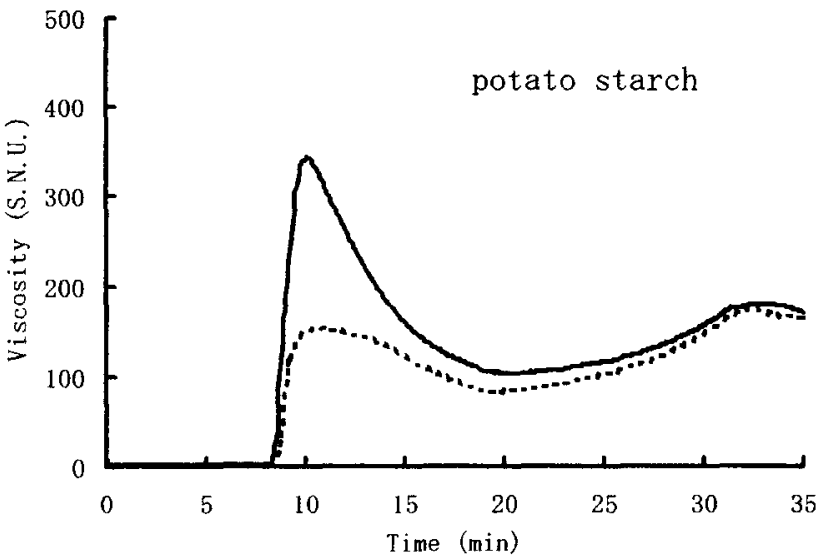

Fig. 4. Effect of $\mathrm{NaCl}$ on starch pasting properties.

- Pasting curve of the starch suspension (Tanikei A6599-4: $3 \mathrm{~g}$; potato: $2 \mathrm{~g}$ ) plus $25 \mathrm{ml}$ of distilled water.

... Pasting curve of the starch suspension (Tanikei A6599-4: $3 \mathrm{~g}$; potato: $2 \mathrm{~g}$ ) plus $25 \mathrm{ml}$ of $0.08 \% \mathrm{NaCl}$.

(Fig. 4) showed that Tanikei A6599-4 starch pasting was only negligibly affected and its stability was maintained, while potato starch pasting, known to be more stable than that of cereal starches, was significantly affected by $\mathrm{NaCl}$, showing a markedly reduced peak viscosity.

\section{Discussion}

The $W x$ gene coding for $W x$ protein (= granule-bound starch synthase) is the principal gene that modifies the amylose content of cereal endosperm, although other genes such as $d u, a e$, or amol were identified in maize, rice, and barley (Creech 1968, Satoh and Omura 1981, Okuno et al. 1983, Yano et al. 1985, Ulrich and Eslick 1978). In diploid cereals, the $W x$ gene dosage in the endosperm of the ordinary type is 3 , and incomplete dominance and dosage effect of $W x$ gene have been reported in maize and rice (Sager 1950 , Okuno et al. 1983, Sano 1984). Since hexaploid wheat has 3 homoeologous $W x$ genes $(W x-A 1, W x-B 1, W x-D 1)$ on group 7 chromosomes (Chao et al. 1989, Nakamura et al. 1993), the $W x$ gene dosage in the endosperm of the ordinary type is 9 in total. Although it had already been reported that the line having 3 null alleles at 1 or 2 of the 3 homologous $W x$ loci, e.g., [null null null, null null null, Wx-D1 Wx-D1 Wx-DI] contains less amylose than the ordinary one (Yamamori et al. 1994, Graybosh et al.1998), the amylose content in a line having 1 or 2 null alleles at each $W x$ locus, e.g., [Wx-AI null null, Wx-BI null null, $W x-D 1$ null null $]$ remained to be determined. We analyzed the amylose content of reciprocal $F_{1}$ seeds in 4 cross combinations (Table 1). In 3 cross combinations, excluding that between Norin 61 and Tanikei H1881, only the $W x-D l$ gene was involved, as in the case of the diploid plants. In the dominance among multiple $W x-D I$ alleles, the $W x-D 1$ allele of Tanikei A6099 partially dominated those of Tanikei A6599-4 and Tanikei H1881, and the allele of Tanikei A6599-4 partially dominated that of
Tanikei H1881. In the cross combination between Norin 61 and Tanikei H1881, $3 W x$ genes were involved, and $F_{1}$ seeds of Norin 61/Tanikei H1881 showed a lower and those of Tanikei H1881/Norin 61 a much lower amylose content than Norin 61, indicating that incomplete dominance and dosage effect of $W x$ genes are evident in wheat regardless of polyploidy.

Compared to the amylose-free waxy lines, Tanikei A6599-4 is unique, as it contains a small amount of amylose and its starch and pollen grains stained dark brown with a $\mathrm{KI}^{-} \mathrm{I}_{2}$ solution (Kiribuchi-Otobe et al. 1998b). The present study showed that at a $6 \%$ concentration, the peak viscosity of Tanikei A6599-4 starch was closer to that of Tanikei $\mathrm{H} 1881$, but the peak viscosity temperature was the same, $94^{\circ} \mathrm{C}$, as that for Norin 61 (Fig. 3). This phenomenon is compatible with the microscopic observation of the swelling process of individual starch granules heated in excess water (Otobe and Kiribuchi-Otobe 1999). They reported that Tanikei A6599-4 starch exhibited morphological changes similar to those of amylose-free wheat, but that its swelling ratio was close to that of nonwaxy wheat.

Yanagisawa et al. (2001) observed that Tanikei A6599-4 contained the same amount of Wx-D1 protein as Tanikei A6099 but with 1 base change from $G$ to A leading a substitution of 1 amino acid from alanine to threonine. Assuming that the $\mathrm{Wx}-\mathrm{D} 1$ protein mutation reduced the starch synthase activity, the reduction of the amylose content is understandable. Such waxy mutants that produce inactive Wx proteins were reported in maize and rice (Echt and Schwartz 1981, Yano et al. 1988). Furthermore, our study showed that the stable hot paste viscosity was also controlled by the mutated $W x-D I$ gene. If the endosperm starch of Tanikei A6599-4 is a chimera of waxy and nonwaxy starch, such a unique pasting property may be explainable, because swollen nonwaxy starch granules with a higher peak viscosity temperature may maintain viscosity 
after waxy starch granules collapsed. Nevertheless, such an assumption can be ruled out because the starch granules of Tanikei A6599-4 uniformly stained dark brown with a potassium iodine and iodide solution (Kiribuchi-Otobe $e t$ al. 1998b) and a simulated 1.6\% amylose mixture of Tanikei H1881 and Tanikei A6099 did not show hot paste stability (Kiribuchi-Otobe et al. 1998b).

It is generally assumed that phosphate-monoester derivatives in starch increase the paste viscosity. Potato starch, which contains a large amount of phosphatemonoesters, is more resistant to heat and shearing than cereal starches, but its hot paste stability is lost when potassium bound to phosphate-monoester is displaced by other cations (Hofstee and de Willigen 1956). Our present study also showed that the peak viscosity of potato starch decreased markedly by the addition of $\mathrm{NaCl}$, whereas the pasting curve of Tanikei A6599-4 starch did not change (Fig. 4). Therefore we consider that the stability of the hot paste viscosity of Tanikei A6599-4 is unrelated to the content of phosphate-monoesters, although we have not measured it. It remains to be determined why the hot paste viscosity of Tanikei A6599-4 is stable. Although the effect of the presence of $1.6 \%$ amylose cannot be ruled out, it is likely that the starch property mostly depends on the amylopectin structure in Tanikei A6599-4. If the mutated $W x-D 1$ gene affects both amylose and amylopectin synthesis, it is of deep interest and deserves further exploration.

\section{Literature Cited}

Chao, S., P.J.Sharp, A.J.Worland, E.J.Warham, R.M.D. Koebner and M.D.Gale (1989) RFLP-based genetic maps of wheat homoeologous group 7 chromosomes. Theor. Appl. Genet. 78: 495-504.

Creech, R.G. (1968) Carbohydrate synthesis in maize. Adv. Agron. 20: 275-322.

Echt,C.S. and D. Schwartz (1981) Evidence for the inclusion of controlling elements within the structural gene at the waxy locus in maize. Genetics 99: 275-284.

Eriksson, G. (1969) The waxy character. Hereditas 63: 180-204.

Graybosch, R.A., C.J.Peterson, L.E.Hansen, S. Rahman, A. Hill and H.Skerritt (1998) Identification of U.S. wheats carrying null alleles at the $w x$ loci. Cereal Chem. 75: 162-165.

Hofstee, J. and A.H.A.de Willigen (1956) Starch. In "Foodstuffs, their plasticity, fluidity and consistency" G.W.S. Bair (ed.), J. Nikuni and T.Isemura (trans.), Asakura Shoten, Tokyo. p. 1-33.

Kiribuchi-Otobe, C., T.Nagamine, T, Yanagisawa, M.Ohnishi and 1. Yamaguchi (1997) Production of hexaploid wheats with waxy endosperm character. Cereal Chem. 74: 72-74.

Kiribuchi-Otobe,C., T. Yasui, T. Yanagisawa and H. Yoshida (1998a) Allelism test of waxy hexaploid wheats from different sources. Breed. Sci. 48: 93-94.
Kiribuchi-Otobe,C., T.Yanagisawa, I.Yamaguchi and H.Yoshida (1998b) Wheat mutant with waxy starch showing stable hot paste viscosity. Cereal Chem. 75: 671-672.

Nakamura, T., M. Yamamori, H. Hirano and S. Hidaka (1993) Identification of three $\mathrm{Wx}$ proteins in wheat (Triticum aestivum $\mathrm{L}$.). Biochem. Genet. 31: 75-86.

Nakamura,T., M. Yamamori, H.Hirano, S. Hidaka and T. Nagamine (1995) Production of waxy (amylose-free) wheats. Mol. Gen. Genet. 248: 253-259.

Oda, S., C. Kiribuchi and H.Seko (1992) A bread wheat mutant with low amylose content induced by ethylmethane sulphonate. Jpn. J. Breed. 42: 151-154.

Okuno, K., H. Fuwa and M. Yano (1983) A new mutant gene lowering amylose content in endosperm starch of rice, Oryza sativa $\mathrm{L}$. Jpn. J. Breed. 33: 387-394.

Otobe, K. and C. Kiribuchi-Otobe (1999) Changes in starch granules trapped and heated using optical tweezers. Starch/Stärke 51: 157-160.

Rohde, W., D. Becker and F. Salamini (1988) Structural analysis of the waxy locus from Hordeum vulgare. Nucl. Acids Res. 16: 7185-7186.

Sager, R. (1950) On the mutability of the $w x$ locus in maize. Genetics 36: 510-540.

Sano, Y. (1984) Differential regulation of waxy gene expression in rice endosperm. Theor. Appl. Genet. 68: 467-473.

Satoh, H. and T.Omura (1981) New endosperm mutations induced by chemical mutagens in rice, Oryza sativa L. Jpn. J. Breed. 31 : 316-326.

Suenaga, K. and K.Nakajima (1989) Efficient production of haploid wheat (Triticum aestivum) through crosses between Japanese wheat and maize (Zea mays). Plant Cell Rep. 8: 263-266.

Ulrich, S.E. and R.F.Eslick (1978) Evidence for assigning the high amylose locus amol of 'Glacier' barley to chromosome 2. Barley Genet. Newslett. 8: 112-113.

Ushiyama,T., T. Shimizu and T.Kuwabara (1991) High frequency of haploid production of wheat through intergeneric cross with teosinte. Jpn. J. Breed. 41: 353-357.

Yamamori, M., T.Nakamura, T.R.Endo and T.Nagamine (1994) Waxy protein deficiency and chromosomal location of coding genes in common wheat. Theor. Appl. Genet. 89: 179-184.

Yanagisawa, T., C. Kiribuchi-Otobe and I. Yamaguchi (1996) Identification of waxy protein in waxy hexaploid wheats. Breed. Sci. 46 (Suppl. 1): 148.

Yanagisawa, T., C. Kiribuchi-Otobe and H. Yoshida (2001) An alanine to threonine change in the Wx-D1 protein reduces GBSS I activity in waxy mutant wheat. Euphytica 121: 209-214.

Yano, M., K.Okuno, J. Kawakami, H. Satoh and T.Omura (1985) High amylose mutants in rice, Oryza sativa L. Theor. Appl. Genet. 69: 253-257.

Yano, M., Y.Suzuki, K. Okuno and H. Satoh (1988) Gene expression of $w x$ locus in rice. Jpn. J. Breed (Suppl. 1): 404-405.

Yasui, T., T. Sasaki, J.Matsuki and M. Yamamori (1997) Waxy endosperm mutants of bread wheat (Triticum aestivum L.) and their starch properties. Breed. Sci. 47: 161-163. 